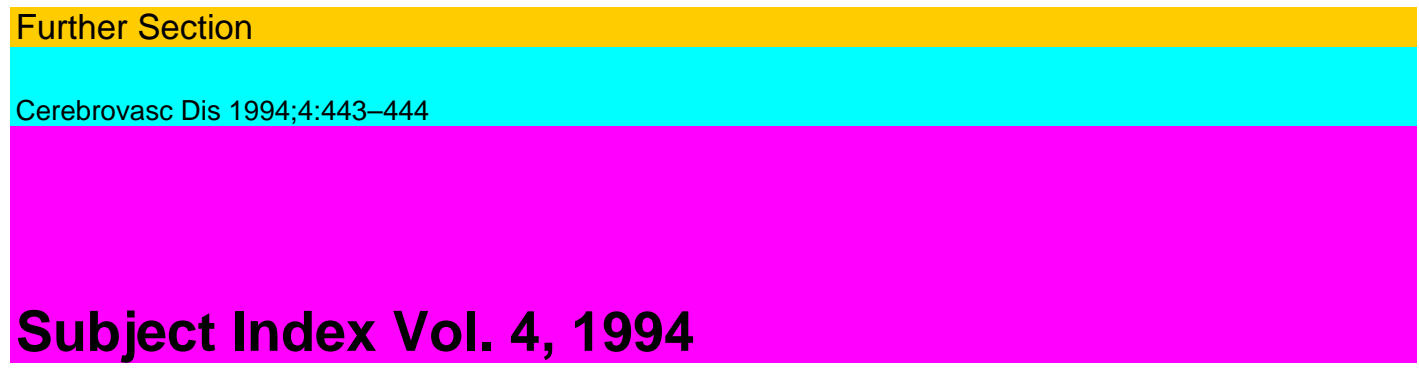

ACE inhibitors 354 Acetazolamide 134 Acute care 432

hemispheric stroke 179

isch(a)emic stroke 189,197,204,385, 398

stroke 3,72

Adult moyamoya disease 106

Alcohol 59

Ambient temperature 359

Aneurysm 329

Angiography 125, 365

Anticardiolipin antibodies 76

Arrhythmias 309

Arteritis 125

Aspirin 157

Atrial septal aneurysms 337

Australia 435

Barthel index 314

- scale 341 Basal ganglia 83 Basilar aneurysm 371

- artery 407 Behavioral symptoms 44 Blood pressure management 385 Brain infarction 197,437

- ischemia 26

- oedema 412 Brainstem infarction 371

Calcium antagonists 182,189 Cardiac embolism 139

- sources of embolism 20 Carotid endarterectomy 32

- occlusion 175,309

- $\quad$ stenosis 12,417 Carpopedal spasm 106 Case fatality 432 Centrum ovale 83

Cerebral blood flow 287,309,354

- - volume 287

- embolism 152

- h(a)emorrhage 121,437

- hemisphere 179

- infarction 66, 92, 106,412

- ischemia 157,280

- stroke 57,421 Cerebrospinal fluid 125 Cerebrovascular disease(s) 12,83,139,152,

$265,337,365,402$

- disorders 51,72, 139,398,428,432

- reserve capacity 134

Cholesterol 417

Cine-computerized tomography 325 Climate 373 Clinical outcome 101 CNS-1102 26 Collateral flow 32

Computerized tomography 12 Congenital heart disease 428 Cranial computerized tomography 20

Decompressive surgery 385

Dejerine-Roussy syndrome 55

Dementia 44,287

Dependency 314

Diagnosis 57,365

Diet 59

Diffusion imaging 3

Disability 314,344,421

Dissection 88

Drugs 432 
Dyslipidemia 92

Echocardiography 139,325

EEG, stroke 96

Elderly 146

Emboli monitoring 393

Embolism 38,428

Enalapril 354

Epidemiology 344

Epilepsy 96

Epinephrine 398

Essential hypertension 354

Extracranial large-artery disease 20

- $\quad$ vertebral artery 88

Fast imaging 3

Gadolinium-DPTA 12 Genetic polymorphism 298 Glucose level 66 Glutamate 115

Haemorrhagic transformation 412 Handicap 314 Helicopter service 431 Hemorrhagic complications 101 Heparin therapy 101 Hexamethylpropyleneamine oxime 354 Horizontal gaze palsy 51 Hospital referral 72

- $\quad$ services 344Hypercapnia 134Hyperperfusion 32

Impairment 314

Incidence, stroke 435

Infarction 179

Internal carotid artery dissection 170

Intracerebral pressure 385

Intracranial dissection 170

Intravenous administration 204

INWEST 204

Isch(a)emic cerebral hemisphere stroke 179

- cerebrovascular disease 298

- $\quad$ stroke 163,182,359

Lacunar infarction 12,83,265 Lateral geniculate body infarct 55 Leukoaraiosis 287 Leukoencephalopathy 294 Lipohyalinosis 121 Lipoprotein 92 Lipoprotein(a) 298 Lupus anticoagulant 76

Magnetic resonance angiography 3,273, 365

- - imaging 12,125,265,273,294,

325 Management, stroke 430 Mechanical prosthetic heart valves 393 Memory disturbances 44 Meta-analysis 197 Microspheres 309 Middle cerebral artery dissection 170

- $\quad$ - - stenosis 273Minor and trace elements 412Morbidity 66

Mortality 66,402 Moyamoya disease 329

Neurocysticercosis 125 Neuronal protection 115 Nimodipine 179, 182,189,194,197,

204 NMDA antagonist 26 Non-Japanese 106 Norepinephrine 398

Old age 402

Oxford Handicap Scale 314

Particle-induced X-ray emission 412 Pathogenesis, stroke 435 Perfusion imaging $3 \wedge$ Pernpartal cardiomyopathy 325 Physical exercise 59

443

Platelet aggregation 163

- factor 4163

- secretion 163 Pontine hemorrhage 51 Population-based studies 344 Predisposing

factors 359 Prevalence 421

Primary antiphospholipid syndrome 76 Prognosis 44,66, 344 ProteinS 304

Pseudoxanthoma elasticum 294

\section{Questionnaire 57}

\section{Radiculomedullary artery 88}


Randomized controlled trial(s) 204,314

Rankin scale 341

Rats 26

Recovery 314

Rehabilitation 146

Research 435

Risk factors 402,417

Saudi Arabia 402 Self-reported disease 57 Serotonin 163

Single photon emission computed tomography 354 Small deep infarcts 121

- vessel disease 121

Smoking 59 Sneddon's syndrome 76 Spectroscopy 3 Spinal cord ischemia 88

- nerve compression 88Standardization 421

Stroke 38, 83, 96, 115, 139,146,157,194,

287, 304, 325, 337,344,417,435 -, cost 430

- model 309

- mortality 437

- outcome 314,341

- prevention 59,431 -, progression 101

- scales 197

- subtypes 337

- treatment 182

- units 432 -, young 170

Subarachnoid hemorrhage 170,329

Telephone interview, stroke outcome assessment 341 Temperature 66 Temporal distribution 359 Thalamic infarction 44,55 Therapy 115, 189 Thrombolytic therapy 385 Thrombosis 407 Time factors 72

Tomography 407

-, emission computed, single-photon 280 Transcranial Doppler sonography 32,134, 354,393

- - ultrasound 273

- duplex sonography 371 Transesophageal echocardiography 38,139 Transient

ischemic attack(s) 139,373 Treatment 435

Trial 194 Tuberothalamic artery 44

Ultrasonics 152

Validity 57

Vascular risk factors 121 Ventricular pacing 309 Visual-field defect 55

Watershed cerebral infarction 175 White matter disease 294 Women 157

X-ray 407

- computed tomography 125

Young stroke patients 304 
